\title{
HIPERPLASIA SUPRARRENAL FETAL: SU RELACION CON LA TOXEMIA
}

\author{
RESUMEN DEL LIBRO DE WILLIAM BENTON PATTERSON
}

\author{
Dr. Hugo Guevara Jaramillo
}

Especialista del Departamento de Toxemias, I.M.I. Bogotá.

Numerosos estudios se han hecho para dilucidar la causa de la Toxemia. Parece que las Glándulas suprarrenales juegan un factor importante $y$ por ello se ha intensificado el estudio de la Corteza Suprarrenal y sus hormonas.

A - Patogenia de la nutrición embrionaria. Debe recordarse el normal intercambio hormonal entre la madre y el feto para comprender cómo el metabolismo fetal actúa independientemente del de la madre y utiliza sus propias hormonas. En las formas de vida bajas tales como los anfibios, el huevo es fertilizado fuera del cuerpo materno y se desarrolla hacia embrión. A medida que se avanza en la escala, el proceso de reproducción y fertilización, se hace internamente.

$B$ - Conservación y reactivación de los genes. GURDON, demostró que el cromosoma de los genes que no se utiliza en el desarrollo de una célula específica, no se pierde totalmente y puede ser reactivado. El componente del Citoplasma determina la función de una célula y entonces cambiando estos componentes, puede cambiarse la función de la célula.
C - Malignidad producida por dascquilibrio hormonal. La HCG, desempeña un papel importante en el embarazo normal, en la Toxemia y en la producción de tumores Trofoblásticos, por lo cual se debe investigar el papel que pueda jugar el desequilibrio hormonal en dichos tumores. Es importante su estudio en la Toxemia porque en ella se hallan amplias fluctuaciones en la producción hormonal de madre y feto. Los disturbios hormonales prolongados, como lo es un exceso de Hormonas Tróficas, en combinación con Virus pueden desarrollar el Tumor en varios órganos. Al principio el exceso de hormonas puede producir solo Hipertrofia e Hiperplasia del tejido; en el siguiente estado y siendo más alta la concentración hormonal resultaría el desarrollo de adenomas en el tejido y posteriormente puede desarrollarse malignidad del mismo tejido.

Corteza Suprarrenal en el feto de 9 meses. El peso de las suprarrenales de los recién nacidos varía enormemente. BENNER reporta un promedio de peso de 10 gramos. Algunos recién nacidos tienen unas suprarrenales muy pequeñas mostrando diferentes grados de involución. Estos niños pue- 
den dividirse en varios grupos: unos de ellos murieron por trauma Obstétrico, otros fueron monstruos anencéfalos y con problemas hipofisarios y por último otros niños cuyas madres fueron sometidas durante el embarazo a tratamientos con Cortisona o sus derivados.

\section{Involución de la Corteza Suprarre-} nal después del nacimiento. Después del nacimiento la Corteza Suprarrenal presenta una degeneración rápida con necrosis y absorción; esto ocurre tanto en los prematuros como en los niños a término. Ocasionalmente la $\mathrm{Hi}$ perplasia de la corteza suprarrenal del feto no involuciona después del nacimiento y permanece como hiperplasia suprarrenal (corteza) congénita. Numerosos estudios han demostrado que el crecimiento normal de la corteza suprarrenal depende de una Hipófisis normal. La Hipofisectomía intrauterina en ratas y conejas está seguida de atrofia suprarrenal; inyecciones de ACTH a estas ratas y conejas previenen esta atrofia. De esto se deduce que el ACTH materno no atraviesa la placenta en cantidades apreciables.

\section{Absorción de Hormonas fetales. VI-} LLE, al tratar sobre el metabolismo en la Placenta ha demostrado que las moléculas grandes como las Proteínas, Polisacáridos, lípidos, Acido Nucleico, etc. no pasan la barrera placentaria; las moléculas pequeñas como los Aminoácidos, Monosacáridos, Gliceroles etc., pasan fácilmente la barrera placentaria, y aparecen en altas concentraciones tanto en la sangre materna como fetal. Ciertos autores han demostrado un aumento progresivo en la excreción de 17 cetosteroides en el embarazo de mujeres con Enfermedad de Addison. Varios niños nacidos de madres diabéticas presentan hiperplasia de los Islotes de Langerhans y poco después de nacer, algunos de ellos presentan una enfermedad caracterizada por cianosis, edema y aumento progresivo de la Disnea; así mismo presentan hipoglicemia severa. FlSHER y HOBEL estudiaron la Tiroxina libre en el suero. La Tiroxina y la Tirotropina en la sangre materna y fetal, encontrando que las concentraciones de Tiroxina en el suero tenían igual nivel tanto en la sangre materna como fetal. Sinembargo las concentraciones de Tiroxina libre y Tirotropina estaban más elevadas en la sangre fetal.

\section{Niveles Hormonales en el embarazo}

a) Gonadotropina Coriónica. En el ser humano tan pronto comienza el embarazo la HCG aparece en el suero y en la orina de mujeres embarazadas alcanzando su mayor nivel alrededor de la 10a semana para decaer notoriamente entre las semanas 17 a 19 y permanecer estable de allí en adelante hasta la semana 37 , a partir de la cual hay un ligero aumento.

b) Somatotropina. Esta hormona de carácter proteico se encuentra en la placenta, el suero y la orina durante el embarazo.

Parece ser de origen Placentario y tiene cierta actividad Lactogénica. Está presente en el Sincitiotrofoblasto. También está demostrado que se encuentra en mujeres con Tumores Trofoblásticos. Se halla presente desde el 12 . día de gestación continuando en aumento durante el embarazo con valores mínimos de $1 \mathrm{mcgr} . / \mathrm{ml}$. a 14.5 mcgr. $/ \mathrm{ml}$. en el tercer trimestre de embarazo.

c) Progesterona. Durante el embarazo normal hay un aumento gradual de la progesterona plasmática y de la excreción urinaria de su metabolito el Pregnandiol. Su máximo aumento es en las últimas 4 semanas y llega a cifras de $120-140 \mathrm{Ng} / \mathrm{ml}$. El Pregnan- 
diol alcanza niveles de $45 \mathrm{mg}$. en las 24 horas durante las últimas 4 semanas.

d) Estrógenos. Los niveles séricos de estrógenos aumentan lentamente al principio del embarazo y alcanzan su máximo en el último trimestre. Durante el embarazo normal la excreción de estrona y estradiol se eleva ligeramente, no así el estriol que aumenta más.

e) Tiroxina. Durante el embarazo hay un aumento de la función del Tiroides con aumento del metabolismo basal. La lodoproteinemia se eleva y esta eliminación es debida a un aumento de la fracción de Tiroxina.

f) Cortisol. Es el que más se secreta por las suprarrenales. Hay un aumento progresivo del cortisol durante el embarazo; varía entre 12.5 mcgrs. al principio y 35 a 40 mcgrs. en el tercer trimestre.

g) Aldosterona. La secreción normal en mujeres no embarazadas varía entre 50 a 250 mcgr./día. Durante el embarazo hay un gradual aumento el cual comienza alrededor de la 15 a semana llegando a cifras de $1.625 \mathrm{mcgr}$ /día al final de la gestación.

Corteza Suprarrenal en Pacientes Toxémicas. WHITELY y STONER estudiaron las suprarrenales de 3 pacientes que murieron por Toxemia y las compararon con las de 11 mujeres que murieron de enfermedad sistémica severa, 2 de ellas con Síndrome de Cushing y 4 después de tratamiento con ACTH. Se encontró una pérdida de lípidos y cambios en la zona fasciculada. Comprobaron que en la Toxemia había un notorio aumento de los esteroides urinarios en relación con el embarazo normal; así mismo concluyeron que había una correlación definida entre el aumento de la actividad secretora de la zona fasci- culada y el aumento de la excreción de esteroides en la Toxemia.

Como regla general, la excreción de esteroides tiende a ser más baja en la Toxemia que en el embarazo normal. TANGCHAI estudió las Suprarrenales de 8 pacientes muertas por toxemia y encontró hemorragias corticales en 4 casos. Las células se hallan más alargadas, con vacuolas difusas e invadidas de gránulos. Así mismo apreció células binucleadas con hipercromatismo nuclear. En las zonas fasciculada y reticular se encontró con gran frecuencia el pigmento lipocromo. Se estudió la suprarrenal de 33 pacientes muertas por Eclampsia y una tercera parte de ellas presentó hemorragia y necrosis que destruyó el $50 \%$ de la capa cortical.

\section{Evidencia Clínica de la existencia de Hormona Luteotropa Suprarrenal}

a) Aumento de la Gonadotropina luego de la estimulación por ACTH. SOHVAL Y SUFFER trataron con ACTH 22 pacientes con diferentes enfermedades y encontraron que en 9 había aumento de la excreción de Gonadotropina.

b) Aumento de la Gonadotropina después del Stress. Los mismos autores estudiaron la excreción de Gonadotropina en 18 pacientes que habían sido sometidas a Cirugía con anestesia general y en 8 de ellas hallaron aumento de la Gonadotropina que se prolongó hasta por 4 días.

c) Aumento de la Gonadotropina en el Síndrome de Cushing. McMILLAN y CUY reportaron un caso de $\mathrm{S}$. de Cushing con reacción de Friedmann positiva en una mujer con Tumor de Hipófisis. La R. de Friedmann se negativizó y los síntomas desaparecieron luego de la denervación de ambas cápsulas suprarrenales. 
d) Aumento de la Gonadotropina coexistente con Tumor benigno de la Corteza Suprarrenal. McFADZEN reportó un caso de tumor benigno de la Corteza Suprarrenal en un hombre con feminización y $R$ de Friedman positiva. Después de 20 días de extirpado el tumor la $R$. de Friedmann se negativizó.

Aumento de la Gonadotropina con Tumor Maligno de la Corteza Suprarenal. CHAMBERS reportó un caso de Carcinoma primario de la Corteza Suprarrenal con reacción de Friedmann positiva.

Patología Placentaria en la Toxemia. En la Toxemia, lo mismo que en las enfermedades de alto riesgo, la degeneración de la placenta parece que es debida a un proceso progresivo de oclusión vascular. Estudios recientes demuestran que además de la proliferación trofoblástica se encuentran procesos de: endarteritis, fibrosis de las vellosidades y necrosis fibrinoide. El infarto de la placenta no es tan frecuente como antes se creía. La desintegración del tejido placentario ocurre por 2 procesos diferentes: el primero cuando hay endarteritis y oclusión de los vasos arteriales que suplen de sangre los capilares de las vellosidades; las vellosidades se degeneran y pueden servir de nidos para las formaciones de fibrina; estas masas de fibrina pueden hialinizarse, organizarse, degenerarse y se encuentran frecuentemente infartos placentarios. El segundo proceso ocurre cuando hay endarteritis y oclusión del sistema arterial que suple uno o más cotiledones de la placenta, los cuales se degeneran aunque no tan rápido como otros tejidos debido a que la zona infartada recibe nutrición por la sangre materna a través de los espacios intervellosos. El aumento de la proliferación trofoblástica que ocurre des- pués de la degeneración vellosa parece ser un fenómeno que ocurre cuando las vellosidades no tienen suficiente epitelio para producir la progesterona que se requiere para mantener el embarazo.

Lesiones Vasculares y Renales en la Toxemia. TANGCHAI estudió los cambios morfológicos en 8 pacientes que murieron por Eclampsia y encontró que había una hiperplasia de la capa media en las pequeñas arteriolas y arterias de todo el cuerpo que incluía aún: pulmones, hígado, hipófisis, suprarrenales etc. La capa íntima presentaba disminución de su espesor y las células endoteliales eran más grandes. Degeneración fibrinoide de las paredes vasculares y formaciones intravasculares de fibrina se encontraron frecuentemente. A todos estos cambios se agregó una vasoconstricción arteriolar marcada; la capa basal de los glomérulos estaba engrosada, los glomérulos isquémicos, aumentados de tamaño y el espacio glomerular obliterado. Precipitados de fibrina periglomerular se encontraron con gran frecuencia. Las células eran grandes, pálidas, vacuoladas y casi totalmente desprovista de gránulos, sus núcleos grandes y vacuolados y algunas con marcado hipercromatismo. Las células de la mácula densa tenían citoplasma pálido y algunos de sus núcleos eran más grandes y más pálidos de lo normal. ALTCHECK practicó 76 biopsias renales en pacientes con Toxemia y describió 4 tipos de lesiones: 1\% Lesión glomerular. 2\% Hiperplasia de células yuxtaglomerulares. 3․ Lesión del Asa de Henle. 4․ Espasmo de las arteriolas aferentes. El glomérulo está aumentado de tamaño y pálido. La cápsula de Bowman con numerosos nódulos de fibrina así como precipitados de proteínas. El epitelio del asa de Henle con severos cambios degenerativos. Las arteriolas 
aferentes del glomérulo tenían un vasoespasmo acentuado con disminución de la luz intravascular y edema de sus paredes. Todas las lesiones desaparecieron en el post-parto aunque algunas de ellas permanecieron hasta por 5 meses.

Hormonas y Metabolismo intracelular. Una hormona es una substancia química producida en el organismo y que tiene un efecto específico en la actividad de cierto órgano. Su acción es coordinar y regular el complejo sistema enzimático dentro de la célula sin que ella misma sea componente esencial de este sistema. Selye ha demostrado que glándulas secundarias como las suprarrenales requieren para su funcionamiento hormonal primariamente hormonas hipofisarias pero que la glándula continúa funcionando aún en animales hipofisectomizados aunque con menos efectividad. Parece ser que los sistemas enzimáticos dentro de las células pueden ser sobreestimulados resultando una función excesiva o pueden ser inhibidos, resultando una disminución de la función, o la reacción bioquímica dentro de la célula puede cambiar por cambios del sustrato lo cual daría como resultado una función diferente.

Cultivos de tejidos placentarios. Los cultivos de tejido placentario han demostrado alguna producción de Gonadotropina. Así mismo cultivos de Mola Hidatidiforme y Coriocarcinoma producen gonadotropina. Tejido placentario ha crecido en el ojo de conejos produciendo respuesta ovárica en el huésped. Así mismo la orina del huésped produce respuesta ovárica cuando se inyecta en un segundo conejo. El tejido placentario crece pobremente en cultivos de tejidos a menos que contengan tejido embrionario o suero de animales embarazadas. Se piensa que el tejido placentario comienza con el Trofoblasto del estado prevelloso que produce HCG y que la función de tal Gonadotropina placentaria es alertar al cuerpo materno de que existe un embarazo. El sitio de acción de esta Gonadotropina sería el Cerebro, probablemente el hipotálamo del cual saldría el estímulo para la $\mathrm{Hi}$ pófisis y otras partes del cuerpo. La función de grandes cantidades de HCG sería servir como hormona trófica para la producción de progesterona en el cuerpo lúteo y más tarde en la placenta una alta secreción de progesterona necesaria para mantener el embarazo normal.

Papel de la progesterona en el embarazo. La progesterona ha sido denominada la hormona del embarazo porque además de su papel precursor en la génesis de varias hormonas esteroides, es necesaria para preparar el endomertio, para la fertilización del óvulo etc. Cuando el embrión es retenido en el cuerpo materno, con el desarrollo de la placenta, el ovario asume una nueva función endocrina en la producción y secreción de progesterona. El cuerpo Lúteo y la progesterona están bajo la influencia de la hormona Luteotropa. En la suprarrenal el colesterol es convertido primero en pregnanolona y luego en progesterona; luego en cortisol y luego en androsterona. El Cortisol también se puede formar de la 17 hidroxipregnenolona. Estrógeno es el producto final de la testosterona formada en el ovario. Entonces se ve que la progesterona juega papel importante en la formación de: cortisol, hidrocortisona, androsterona, testosterona y estrógeno. Si hay una degeneración de la placenta de manera que ésta no pueda producir la progesterona necesaria, es lógico pensar que las glándulas endocrinas son capaces de producirla. Hay muchas evidencias de que esto sucede a nivel de las suprarrenales, sinembargo la suprarrenal aparente- 
mente no es apta para secretar progesterona por largos períodos.

Hormonología del embarazo normal en el primer trimestre. Los investigadores concluyen que la elaboración de HCG comienza 7 a 9 días después de la ovulación y 1 a 2 días después de la implantación del huevo para ir aumentando progresivamente para alcanzar su máximo a las 11 a 13 semanas. La somatotropina se ha encontrado presente en el suero desde el $12^{\circ}$ día de la gestación. La somatotropina es la hormona del crecimiento de la placenta y sus niveles en el suero corren paralelos al crecimiento de la placenta. Se ha localizado en el Sincitiotrofoblasto por Inmunofluorescencia. También por inmunofluorescencia se ha localizado la HCG tanto en el Citotrofoblasto como en el Sincitiotrofoblasto pero en mayores concentraciones en este último. La presencia HCG y HCS en las células trofoblásticas indica que es allí donde se metabolizan y tienen su acción específica y no prueban que allí es donde sean sintetizadas. Durante el embarazo normal, los niveles de progesterona corren paralelos con los niveles de HCG en el plasma. Durante la fase luteínica del ciclo menstrual el nivel de progesterona en el suero alcanza a $15 \mathrm{ng} / \mathrm{ml}$. y alrededor de las 5 semanas de gestación llega a $25 \mathrm{ng} /$ $\mathrm{ml}$. Poco tiempo después de formado el blastocisto, luego de la implantación del huevo, las primeras células trofoblásticas segregan una hormona gonadotrópica que es absorbida por la circulación materna. Esta hormona llega al cerebro donde alerta a los centros nerviosos de la presencia del desarrollo de un embrión en el cuerpo humano y es entonces cuando por estimulación neurohormonal que la hipófisis secreta hormona Luteotropa. Esta L. H. hipofisiaria estimula la glándula suprarrenal que entonces produce hormona gonadotrópica. Esta HCG suprarrenal parece ser la hormona trófica para que el cuerpo lúteo roduzca progesterona. Cuando se necesita mayor cantidad de progesterona para mantener el embarazo y el cuerpo lúteo no es capaz de producirla hay un estímulo a los centros cerebrales que estimulan la hipófisis y finalmente la corteza suprarrenal que ocasiona el aumento de la HCG que incrementa el aumento de progesterona que se requiere para sostener el embarazo.

Poco tiempo después de la 9a semana la función del cuerpo lúteo declina y entonces la placenta toma a su cargo la mayoría de la progesterona que el embarazo necesita. La HCG es una hormona luteotropa como se ha probado por su efecto sobre los ovarios de animales y humanos, estimula las células del cuerpo lúteo para producir progesterona y tiene también una acción sinérgica con la FSH para estimular la producción de estrógenos. Los altos niveles de HCG que se encuentran al principio del embarazo podrían estimular las células de la corteza suprarrenal para sintetizar progesterona y esta estimulación podría conducir a la hipertrofia e hiperplasia de la corteza suprarrenal materna. WHITELY y STONER han demostrado que hay una hiperplasia de la zona fasciculada de la corteza suprarrenal materna al principio del embarazo pero que esta hipertrofia normalmente no progresa.

Etiología de las lesiones vasculares de la placenta. La explicación etiológica de la extensa lesión arterial degenerativa en las placentas de pacientes toxémicas parece ser la misma de la arterioesclerosis en otras partes del cuerpo con la diferencia de que en ésta hay menos variaciones de la presión intravascular. Parece que hay 2 fases en el proceso degenerativo: en 
la primera fase la endarteritis parece ser la lesión predominante que afecta las pequeñas arterias de las vellosidades; en la segunda fase hay una vasoconstricción y degeneración muscular de las paredes de las arterias. BARTHOLOMEW sugiere que estos cambios son debidos a la hipercolesteronemia del embarazo que se presenta usualmente. El colesterol atraviesa la barrera placentaria de madre a feto $y$ viceversa pero es transformado prontamente. Esto parece estar de acuerdo con lo informado por KUPERMANN de que las glándulas endocrinas fetales no dependen del colesterol materno para producir sus esteroides suprarrenales o para la producción de progesterona y estrógenos. Hay un exceso de colesterol en la sangre fetal pero no en la materna. Nosotros pensamos que los cambios arteriales degenerativos son debidos a la hipercolesteronemia fetal secundaria a hipotiroidismo materno subclínico y no al colesterol materno. Alguna condición durante el embarazo podría interferir el metabolismo fetal para aumentar el nivel de colesterol en la sangre del feto y parece ser la etiología causante de la enfermedad arterial placentaria. Los niveles de colesterol en el cordón umbilical fueron elevados en las madres con hipercolesteronemia y fue baja en las madres que tenían niveles bajos.

FISHER y HOBEL encontraron que hay un gradiente de Tiroxina libre $y$ de Tirotropina que va del feto a la madre y demostraron también que hay una gran elevación del nivel de Tirotropina fetal dentro de los $30 \mathrm{mi}$ nutos que siguen al nacimiento y que esto produce un aumento del nivel sérico del PBI dentro de las 24 horas siguientes.

Etiología de las lesiones vasculares renales en la Toxemia y en las enfermedades de alto riesgo. La presencia de un agente vasoconstrictor en la toxemia está universalmente aceptado; este agente causa una vasoconstricción arteriolar generalizada ocasionando hipertrofia e hiperplasia de las paredes vasculares de las arteriolas llegando a ocasionar aún la necrosis fibrinoide de los vasos con disminución de la luz de los mismos. La presión arterial vuelve a sus niveles normales más o menos a las 48 horas después del parto ya que este proceso es reversible, pero el daño renal persiste por meses como lo ha demostrado la biopsia renal.

Angiotensina 11 es el más potente agente vasopresor conocido que ejerce su efecto por acción directa sobre las arteriolas periféricas; es el más poderoso estímulo para la producción de Aldosterona por la zona glomerular de la Suprarrenal. La Angiotensina 11 se deriva de la Angiotensina 1 por conversión enzimática; la angiotensina 1 se forma por interacción de la renina con el angiotensinógeno. La renina es una enzima proteolítica que se forma en las células yuxtaglomerulares. En la Toxemia hay extensos y notorios cambios en las células Yuxtaglomerulares lo cual indica que ellas han sido estimuladas hasta la hiperfunción y en algunos casos casi hasta el agotamiento. Parece que los cambios en las paredes vasculares de las arteriolas y los cambios fisiológicos que resultan del hiperaldosteronismo junto con los cambios en el aparato yuxtaglomerular podrían ser el punto de partida de una superacción y sobreacción de renina angiotensina en la toxemia.

Exceso de renina-angiotensina en la toxemia. Una cantidad excesiva de ACTH estimularía la corteza suprarrenal y pueden causar liberación de grandes cantidades de AMP cíclico; la excesiva cantidad de AMP estimularía las células del aparato Yuxtaglomeru- 
lar para secretar renina que se convertiría en angiotensina. La excesiva cantidad de angiotensina produciría un espasmo arteriolar generalizado que conduciría a la hipertensión y a la necrosis fibrinoide de las paredes de las arteriolas y a su vez estimularía la zona glomerular para producir exceso de Aldosterona.

\section{Explicación de los bajos niveles} plasmáticos de Renina-Angiotensina y Aldosterona en la Toxemia. Para comprender cómo los cambios hallados en la Toxemia pueden ser causados por una superproducción y sobreacción de angiotensina y además teniendo bajos niveles de renina-angiotensina y aldosterona es necesario estudiar el metabolismo del sodio y las interacciones de renina-angiotensina y sodio en el embarazo.

a) Proteínas plasmáticas. Se sabe que las hormonas esteroides están dentro de las proteínas del plasma en ciertas cantidades o proporciones. La fracción libre parece ser la parte activa de la hormona y la fracción incorporada permanece inactiva hasta que puede ser transportada a sitios donde puede ser almacenada 0 metabolizada directamente y esto puede suceder en el hígado. Las hormonas pueden ser almacenadas en el tejido adiposo o permanecer en el líquido extracelular. El cortisol se halla presente en plasma en mucha mayor concentración que cualquier otra hormona; el $10 \%$ está libre y el $90 \%$ mezclado con las proteínas del plasma, un $10 \%$ con albúmina y un $80 \%$ con Transcortin que es una glicoproteína. La concentración de cortisol libre y mezclado aumenta gradualmente en el embarazo alcanzando niveles 3 veces mayores que los normales. La progesterona está mezclada con la albúmina pero lo es más con el transcortin. Durante el embarazo $2 / 3$ de la Aldosterona se ligan a la molécula de albúmina. Debido a que la Aldosterona se fija débilmente a las proteínas del plasma se difunde rápidamente fuera del sistema vascular hacia otros compartimentos. En condiciones normales la mayoría de la Aldosterona se metaboliza en el hígado. Cuando el nivel de hormonas libres aumenta, el nivel de las hormonas ligadas también aumenta.

\section{Metabolismo de Sodio y Aldostero-} na. La mayor parte de las hormonas de la corteza suprarrenal afectan los electrolitos y el metabolismo del agua, pero la principal de ellas es la Aldosterona. Los fluídos extracelulares son ricos en $\mathrm{Na}$. Los altos niveles de progesterona en el embarazo afectan directamente el metabolismo de la Aldosterona. La aldosterona y los mineralocorticoides actúan a nivel celular regulando los electrolitos y el balance hídrico a través de los riñones. La aldosterona estimula la actividad enzimática de la membrana celular obligando a las células a absorber $\mathrm{Na}$, lo cual puede suceder independientemente del K. Las células de los diferentes tejidos responden a los corticoides de manera diferente y la aldosterona no obliga a todas las células a absorber iones de $\mathrm{Na}$. La aldosterona actúa en el túbulo renal para ocasionar la reabsorción de $\mathrm{Na}$ y excreción de $K$. Varios factores influyen en la secreción y metabolismo de la aldosterona; en la insuficiencia cardíaca la administración de $\mathrm{Na}$ ocasiona mayor producción de aldosterona; la hipotensión ocasionada por hemorragia o por otras causas, ocasionaría una mayor cantidad de aldosterona que actuaría para producir una retención de $\mathrm{Na}$ y de líquidos para aumentar la presión sanguínea. Cambios en el $\mathrm{Ph}$ sanguíneo, alteraciones de las proteínas del plasma, enfermedades y tumores de las suprarrenales pueden producir bien sea aumento o disminución 
de la secreción de aldosterona. Las enfermedades renales también afectan el metabolismo de la aldosterona por su efecto sobre el aparato Yuxtaglomerular que produce renina y por el efecto de la enfermedad renal sobre la presión arterial. Desequilibrios hormonales que causan aumento de la secreción de aldosterona y esto es lo que ocurre en la toxemia.

\section{Interacciones de la renina- angiotensina, aldosterona $y$ sodio en la toxemia}

En el 3er. trimestre durante el embarazo normal la secreción de aldosterona es 4 a 5 veces mayor que en la mujer no embarazada. En el embarazo normal una dieta baja en sal, combinada con diuréticos, causa un aumento de la secreción de aldosterona, lo cual parece indicar que el metabolismo de la aldosterona en el embarazo normal está influenciado notoriamente por la administración de $\mathrm{Na}$. $\mathrm{Si}$ a una mujer no embarazada se le dan grandes dosis de aldosterona presentará una marcada retención de $\mathrm{Na}$ y eliminación de $\mathrm{K}$; pero cuando se dan dosis de 1.000 mcgr. diariamente por 10 días a embarazadas normales no hay cambios notorios en la eliminación de $\mathrm{Na}, \mathrm{K}$ y agua. LARAUGH cita experimentos en animales los cuales demuestran que la administración simultánea de grandes dosis de angiotensina y aldosterona produce vasculitis necrotizante tal como sucede en la hipertensión maligna. El mismo autor asegura que: 1? en personas normales la angiotensina causa retención renal de $\mathrm{Na}$ y aumento de la sensibilidad presora; la norepinefrina produce lo contrario. 2 ? la angiotensina estimula la secreción de aldosterona al paso que la norepinefrina la reduce. 3o esta acción orientada del $\mathrm{Na}$ por la angiotensina parece tener un componente renal es- pecífico porque marca un aumento de la presión sanguínea cuando es inducido por angiotensina exógena que conduce a una mayor natruresis que la producida por la Norepinefrina. Estos resultados sugieren que hay un sistema interno de control para la regulación de la presión sanguínea.

Al principio del desarrollo de la toxemia cuando hay un moderado grado de degeneración placentaria, aparece un aumento de angiotensina que estimula la suprarrenal a secretar exceso de aldosterona que ocasionaría retención de $\mathrm{Na}$ y líquidos; ésta combinación de excesos de angiotensina y aldosterona produciría espasmo arteriolar con la consiguiente hipertensión. El exceso de aldosterona causaría exceso de retención de $\mathrm{Na}$ y líquidos que podrían pasar del sistema vascular hacia los espacios extracelulares. El exceso de aldosterona y iones de $\mathrm{Na}$ en el líquido intra y extraccelular podría absorber líquidos y causar edema periférico.

Pacientes con marcado déficit de Progesterona y Toxemia Severa. Como hay un gran déficit de progesterona, la hipófisis se estimula causando el aumento del ACTH y de LH. La LH causa aumento de la HCG suprarrenal que a su vez estimula el tejido trofoblástico para aumentar la secreción de progesterona. Este aumento de la HCG suprarrenal combinado con la HCS produciría botones sincitiales y regeneración trofoblástica lo que ocurre después de la degeneración de las vellosidades. El aumento de ACTH estimula la corteza suprarrenal materna ocasionando aumento de la secreción de cortisol y de aldosterona. El cortisol será sustituído por progesterona y la aldosterona causa disturbios en el metabolismo del $\mathrm{Na}$ con retención del mismo y líquidos en espacios intra y extracelulares. El exceso de AMP cíclico ocasionado por estimulación de 
ACTH estimula el aparato Yuxtaglomerular para secretar exceso de renina que se convierte en angiotensina, la cual produce espasmo arteriolar el cual ocasiona aumento de la presión arterial y vasculitis necrotizante. La vasculitis necrotizante produce endoteliosis en los glomérulos, nefroesclerosis y otros cambios degenerativos en el riñón que interfieren la función renal.

Por el exceso de aldosterona y $\mathrm{Na}$, varios componentes iónicos salen afuera por la presión osmótica alterada. Cuando el nivel del calcio ionizado baja de los niveles normales en los espacios extracelulares, viene la tetania, la cual está caracterizada por aumento de la irritabilidad de todos los músculos, lo mismo que convulsiones tónico-clónicas y espasmos fibrilares; esto combinado con el espasmo de las arteriolas cerebrales parece ser la causa de las convulsiones en la Eclampsia. JEACOCK piensa que la concentración de $\mathrm{Ca}$. en las placentas de pacientes toxémicas es mucho mayor que en las splacentas normales. El embarazo agrava el hiperparatiroidismo; varios casos de éste, con convulsiones y muerte en el puerperio, han sido diagnosticados como Eclampsia post-parto, sin examen de las gl. paratiroides. CARRETERO y cols. demostraron que en el líquido amniótico de algunas pacientes había grandes cantidades de angiotensina.

HCG como hormona trófica. La HCG producida por la suprarrenal tiene una función normal en la fisiología de los ovarios, los testículos y la placenta. El tejido trofoblástico produce otra hormona gonadotrópica cuya función es alertar a los centros hipotalámicos de la presencia de un embrión desarrollándose en el cuerpo. Algunas de estas evidencias son:
1. Ovarios. Sin duda alguna la LH es una hormona trópica para que la suprarrenal produzca HCG y esta es una hormona trópica que controla la producción de Progesterona por los ovarios. La zona reticular de la corteza suprarrenal parece ser el sitio donde se produce la HCG porque ella se hipertrofia luego de la castración de machos y hembras. La orina de las embarazadas contiene un aumento de las hormonas luteinizante y foliculoestimulante lo cual se demuestra por el Test de Friedmann. La HCG es una hormona pura $y$ se ha demostrado que no tiene ninguna acción folículoestimulante, sinembargo siempre se asocia a la hormona folículo-estimulante por lo cual uno puede suponer que la corteza suprarrenal también produce una hormona folículo-estimulante que está bajo control de la FSH hipofisiaria, así como la HCG suprarenal está controlada por la LH hipofisiaria.

2․ Placenta. ZONDECK demostró que la mitad de la HCG inyectada al conejo se destruía a la hora y que a las 24 horas, solo se encontraba el $10 \%$. Solamente el $5 \%$ era excretada por la orina. 7 a 9 días después de la ovulación y 1 a 2 días después de la implantación del huevo, el sincitiotrofoblasto no es más que un sólido cascarón de células que justamente comienzan a formar lagunas que no reciben sangre antes de 3 días. Parece altamente improbable que esta pequeña masa de células trofoblásticas que aún no tiene verdadero contacto con la circulación materna puedan producir grandes cantidades de HCG. Sklow demostró que la HCG en la circulación materna es 25 veces mayor que la encontrada en la sangre del cordón umbilical. Estos hechos sugieren que la placenta absorbe la HCG de la circulación materna y la almacena allí. Los más recientes trabajos de- 
muestran que la placenta no metaboliza el estradiol en estriol debido a la ausencia de la 16 hidroxilasa en la placenta.

3: Tumores trofoblásticos. La HCG de la orina de mujeres con tumores trofoblásticos no difiere de la de la orina de mujeres embarazadas y esto ha sido demostrado por estudios inmunológicos. METCALF revisó algunos principios generales así: a) disturbios prolongados de los reguladores hormonales podrían por sí mismos o en colaboración con tumores vitales, desarrollar tumores en ciertos órganos. b) los tumores desarrollados en tales situaciones inicialmente no son autónomos pero progresan a través de una serie de estados hacia la autonomía, durante la cual la conducta de las células tumorales puede ser profundamente modificada por reguladores normales para cada tipo de célula. Si los ovarios son extraídos y transplantados al bazo, estos tumores eventualmente se desarrollan en los ovarios transplantados; esto sería debido al persistente estímulo ovárico por la gonadotropina hipofisiaria. Las hormonas ováricas que fueron secretadas hacia el bazo, entraron en la circulación portal y fueron inactivadas por el hígado.

Los tumores de los testículos se asocian a menudo con gran producción de gonadotropinas, tal como ocurre en los tumores de la placenta. Las células del tumor de LEYDIG se asocian con aumento de la producción de hormona folículo-estimulante y los seminomas y el epitelioma mixto pueden asociarse con cantidades de hormona folículo-estimulante 0 de ambas hormonas.

Cuando los tumores testiculares son extirpados antes de que haya metástasis, las hormonas gonadotrópicas continúan excretándose en gran- des cantidades y los pacientes no muestran signos de enfermedad. Tumores de tejido trofoblástico, Mola, Corioadenoma, Coriocarcinoma etc. se asocian con alta secreción y excreción de gonadotropina y el descenso en el nivel de las mismas es signo de mejoría luego de extraer el tumor. Sinembargo no es raro encontrar niveles altos de gonadotropina lueg 0 de evacuar la mola y aún luego de histerectomía total. Varios casos de metástasis fatales han tenido mínimos niveles de HCG lo cual se explicaría por la autonomía del tumor.

\section{Resumen y Conclusiones}

1: La hiperplasia corticosuprarrenal del embrión pequeño y del feto parece ser debida al efecto luteotrópico de los altos niveles de HCG en las células corticales que sintetizan progesterona.

$2^{\circ}$ La hiperplasia de la cortical en fetos mayores parece debida al estímulo hipofisiario fetal que resulta cuando la madre absorbe cortisol del feto para reemplazar la deficiente producción de progesterona placentaria.

3: La degeneración placentaria en la primera mitad del mebarazo parece que es debida a la endarteritis oclusiva causada por la hipercolesterolemia que acompaña estados metabólicos fetales que son secundarios a enfermedades y desnutrición materna.

4. Parece existir 2 hormonas gonadotrópicas coriónicas además de las gonadotropinas hipofisiarias Una de ellas se produce en el tejido coriónico y su función es alertar al organismo materno de la presencia primero de un embrión y luego de un feto en el organismo materno. La otra producida por la corteza suprarrenal y pare- 
ce que su función es servir como hormona trópica de la placenta, ovarios y testículos. Durante el embarazo parece que es la HCG suprarrenal la que regula la producción de progesterona por el cuerpo lúteo y la placenta.

5: La HCG suprarrenal parece ser la hormona trópica de los tumores Trofoblásticos. En los primeros estados de formación de dichos tumores ellos son independientes del exceso de HCG suprarrenal. En los últimos estados coom en otros tumores hormonale,s llegan a ser independientes - autónomos y pueden crecer sin un exceso de HCG suprarrenal.

6: La alta concentración de HCG en el primer trimestre parece cleberse a estímulo negativo causado por la inhabilidad del cuerpo lúteo para producir suficiente cantidad de progesterona requerida por el embarazo. Este estímulo actúa a través del eje hipotálamo, hipófisis, corteza suprarrenal que a su vez estimula la placenta para que secrete la progesterona necesaria. El nivel plasmático de progesterona desciende durante el segundo mes de embarazo, lo que indicaría el agotamiento del cuerpo lúteo; de allí en adelante hay un aumento gradual hatas el 9: mes de embarazo. La secreción de HCG es mayor alrededor de la 9a semana cuando la secreción de progesterona es más baja. Cuando la progesterona aumenta hay una caída brusca de la HCG, luego de la secreción de la una corre paralelamente a la de la otra.

7: Cuando hay una extensa degeneración de la placenta y ésta no puede producir la cantidad de hormona que se requiere para el embarazo, habrá entonces un estímulo de la hipófisis materna para secretar ACTH y LH. La LH estimula la suprarrenal para se- cretar más HCG suprarrenal que a su vez estimula el tejido trofoblástico para que secrete más progesterona. El ACTH estimula las células de la corteza suprarrenal que sintetizan la Pregnenolona y la progesterona por conversión del cortisol.

8: Cuando la deficiencia de progesterona es severa, la corteza suprarrenal se agita porque debido a que su tamaño es muy pequeño comparado con la placenta y no sería apta para secretar suficiente cortisol para reemplazar el déficit de progesterona. La corteza suprarrenal estando casi agotada pero luego de un estímulo puede producir aumento del AMP cíclico cuyo exceso estimula las células renales y Yuxtaglomerulares a secretar excesiva cantidad de renina que sería convertida en angiotensina y el exceso de étsa causaría espasmo arteriolar generalizado que llevaría a la hipertensión y a la necrosis fibrinoide de las paredes de los vasos, estimulando también la zona glomerular de la corteza suprarrenal para producir exceso de Aldosterona que ocasiona retención de iones de Sodio.

9: La diabetes gestacional puede desarrollarse cuando hay un exceso de AMP cíclico luego de una deficiencia de progesterona causada por degeneración placentaria. La función del AMP cíclico es como segundo transportador o mensajero para que la epinefrina y el glucagón produzcan glicógénesis en el hígado; así mismo su exceso puede causar aumento del azúcar circulante en la sangre. El resultado final de una excesiva cantidad de AMP cíclico sería aumentar la Glicogenolisis y disminuir la acción de la Insulina produciendo una Diabetes gestacional. 